\title{
Efficient Dynamic Spectrum Sharing Through Rate Compensation and Spectrum Handoff
}

\author{
Adisorn Lertsinsrubtavee, Naceur Malouch and Serge Fdida \\ Laboratoire d'Informatiqe de Paris 6 (LIP6) \\ Université Pierre et Marie Curie (UPMC) \\ Paris, France \\ Email: \{Adisorn.Lertsinsrubtavee, Naceur.Malouch, Serge.Fdida\}@lip6.fr
}

\begin{abstract}
In this work, we propose a heuristic for dynamic spectrum sharing in cognitive radio networks. The concept of rate compensation is introduced so that cognitive radio users are able to achieve their rate requirement by performing adequately spectrum handoffs. Indeed, performing spectrum handoff can increase the achieved rate obtained by moving from unavailable channels to available ones. However, handoffs should also be reduced to decrease handoff delays and access contention in the network which can in turn impact the achieved rate.
\end{abstract}

\section{INTRODUCTION}

The scarcity of the available spectrum in wireless networks is rising new challenges related to the design of new network paradigms for wireless communication. The emerging cognitive radio technology is a promising solution that allows secondary users (SUs) temporarily occupy spectrum bands or white spaces that are unused by their primary users (PUs). One of the main challenges is the ability to share the available spectrum dynamically and efficiently among different SUs in order to satisfy their rate requirements.

Different tools including game theory have been applied in several works (e.g., [1]) in order to achieve static spectrum allocations, where SUs are assigned to some portion of the spectrum. However, in a dynamic environment where the total available capacity varies over time, the static allocation may not be sufficient to reach the rate required by the user. Therefore, spectrum handoff can achieve higher capacity utilization by allowing the user to switch from unavailable channels to available ones. Dynamic spectrum sharing is addressed in several works such as [2], [3]. The spectrum allocation is computed instantaneously based on the current state of the spectrum, such as the number of available channels. Consequently, re-assignment is requested when the state of the spectrum is changed which refers to the arrival or departure of primary and secondary users. Intuitively, some SUs may perform spectrum handoff to collect as much available resources as possible from different channels so that their requirement can be achieved. However, unnecessary spectrum handoffs can cause service degradation due to handoff delays and also increasing contention as discussed in [4].

A global spectrum sharing strategy is computed in our previous work [5] where we assume global knowledge of

Part of this work was supported by the grant ANR-10-VERS-005-03 channel states including their availability during the total communication period. Obviously, the allocation can be optimized globally and the number of spectrum handoff is decreased significantly, since SU may not perform spectrum handoff immediately when one of its current channels is not available. This is because it can compensate its lost rate when the current channel becomes available again, or when it moves later to another available channel. Nevertheless, this global optimization can be complex and not scalable with the number of users. Besides, the allocation and the spectrum handoff sequence provided by the solver software can not be converted to a distributed solution of the dynamic spectrum sharing problem.

In this paper, we describe how to design an efficient heuristic for spectrum sharing which can provide the required rate for all users while decreasing the number of spectrum handoffs. The objective is to attempt to reach the global optimization performance through a practical dynamic spectrum sharing.

\section{DyNAmic SPECTRUM Sharing MOdel}

Consider an infrastructure-based CRN with a total of $N$ secondary users (SU) and $M$ licensed channels available for opportunistic spectrum access. Each SU is equipped with $n$ wireless interfaces and able to transmit over multiple channels simultaneously. The system model is illustrated in Fig.1.

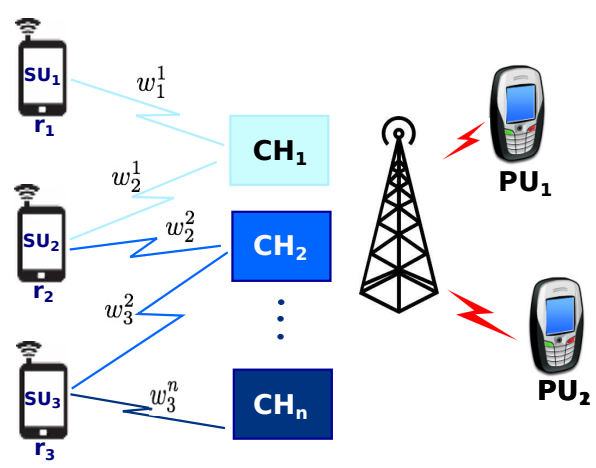

Fig. 1. A spectrum sharing model for cognitive radio networks

The total capacity of channel $i$ is denoted by $b w^{i}$. Each user $j$ has a different rate requirement $r_{j}$, which can be considered also as the user weight for sharing the available bandwidth. At a given time, each SU is allocated a fraction of bandwidth 
from each channel. Denotes by $w_{j}^{i}$ the allocated fraction for SU $j$ over channel $i$, where $w_{j}^{i} \in \mathbb{R} ; 0 \leq w_{j}^{i} \leq 1$. If $w_{j}^{i}=0$, then SU $j$ is not tuned to channel $i$ and thus is not transmitting over this channel. The role of the CR base station is to find the appropriate weights over time which implies also determining the sequence of spectrum handoffs. In addition, we assume that channel availability periods are known in advance through for instance regional databases [6].

\section{Rate Compensation And Spectrum HandofF}

According to the dynamic spectrum nature in the network, spectrum handoff can enhance the achieved rate by switching from unavailable channels to available ones. However, waiting at the current unavailable channel in an attempt to compensate later the lost rate is also useful since it reduces the number of spectrum handoffs, which in turn can increase the achieved rate. Thus, we propose a rate compensation mechanism by introducing the use of a reservation period. A fixed allocation will be used during this period without any handoff. If some SUs do not achieve their rate, they can compensate in the next reservation period by allocating to them a larger rate so that the average rate at the end of their connection meets the requirement. We denote by $T_{r}$ the reservation period. The allocation can change only at the end of each period as illustrated in Fig. 2. Hence, during unavailable periods inside $T_{r}$, spectrum handoffs are not performed.

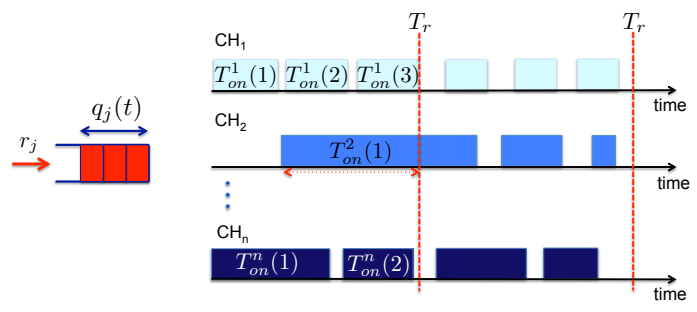

Fig. 2. The reservation period

At the end of $T_{r}$, some SUs may not receive a sufficient bandwidth to send all their packets. This is reflected in their transmission queue $q_{j}(t)$. Thus, the rate of SUs should be related to the number of packets in the queue in order to compensate the lost rate during the former $T_{r}$. The total number of packets to be sent in the next period $T_{r}$ can be obtained as follows:

$$
P_{\text {send }_{j}}\left(T_{r}\right)=r_{j} \cdot T_{r}+q_{j}(t)
$$

To allocate the available bandwidth properly, first, we compute the total capacity $P_{\text {total }}^{i}\left(T_{r}\right)$ of channel $i$ when it is selected to $S U_{j}$ :

$$
P_{\text {total }}^{i}\left(T_{r}\right)=\left\{\begin{array}{cl}
\frac{\sum_{l=1}^{k} T_{o n}^{i}(l) \cdot b w^{i}}{P_{s i z e}}, & i \in C H_{j} \\
\frac{\sum_{l=1}^{k} T_{o n}^{i}(l) \cdot b w^{i}}{P_{s i z e}}-\left\lceil\frac{H O_{\text {delay }}}{P_{\text {size }}}\right\rceil, & i \notin C H_{j}
\end{array}\right.
$$

where $T_{o n}^{i}$ is available period of channel $i, k$ is the number of available periods in $T_{r}, P_{\text {size }}$ is the size of transmitting packets and $b w^{i}$ is the bandwidth of channel $i$. To consider the overhead of spectrum handoff, the handoff delay $H O_{\text {delay }}$ is included when $S U_{j}$ selects a channel that is not in the current set of active channels $C H_{j}$. Now, $w_{j}^{i}$ is computed proportionally to the ratio between $P_{\text {send }}\left(T_{r}\right)$ and $P_{\text {total }}^{i}\left(T_{r}\right)$. In addition, the service satisfaction of SUs can be measured as the ratio of achieved rate $a_{j}$ to the rate requirement $r_{j}$. Consequently, SU who has the lowest satisfaction ratio should have the priority to select the channels to use in the next period in order to compensate from the insufficient allocated rate of previous period. The number of wireless interfaces $n_{j}$ should be also considered in determining the priority of selecting the channels. Indeed, the less $n_{j}$, the less opportunity to achieve the required rate. Since, the total required rate can only be transmitted to few specific channels which is hard to find, especially when this rate requirement is large.

Clearly, the number of spectrum handoffs and the ability of rate compensation can be controlled through the $T_{r}$ parameter. For instance, if $T_{r}$ is very large or equals to the total connection period, the allocation can be static where spectrum handoff will not be performed and the compensation is not considered. On the other hand, when $T_{r}$ is very small, the allocation is more dynamic, thus with more and faster compensation possibilities to achieve the rate requirement. Nevertheless, the number of handoffs can be unnecessarily large.

\section{ONGOING AND FUTURE WORK}

Currently, we are investigating the optimal value for $T_{r}$ which depends mainly on the traffic load and the availability patterns of different channels. Also, by assessing the quality of the allocation of the next period, we can adjust dynamically the size of $T_{r}$. For example, when the availability of the channels do not change anymore, then after few spectrum handoffs, all SUs can compensate and the allocation becomes static, which mean a longer $T_{r}$ is used. Besides, this approach can be implemented in a distributed manner where every user computes locally $T_{r}$ and triggers spectrum handoffs accordingly. Finally, a long term knowledge of channels availability may not be accessible to all SUs. Thus, the heuristic should be adapted to use only previous rate measurements.

\section{REFERENCES}

[1] N. Nie and C. Comaniciu, "Adaptive channel allocation spectrum etiquette for cognitive radio networks," in IEEE International Symposium on New Frontiers in Dynamic Spectrum Access Networks (DySPAN), 2005, pp. 269-278.

[2] W.-Y. Lee and I. F. Akyldiz, "A spectrum decision framework for cognitive radio networks," IEEE Trans. Mobile Computing, vol. 10, pp. 161-174, Feb 2011.

[3] D. Xu, E. Jung, and X. Liu, "Efficient and fair bandwidth allocation in multichannel cognitive radio networks," IEEE Trans. Mobile Computing, vol. 11, no. 8, pp. 1372-1385, Aug 2012.

[4] A. Lertsinsrubtavee, N. Malouch, and S. Fdida, "Controlling spectrum handoff with a delay requirement in cognitive radio networks," in ICCCN, Aug 2012.

[5] — , "Interaction between spectrum handoff and sharing in multi-channel cognitive radio networks," Tech. Rep., Oct 2012. [Online]. Available at: http://www-rp.lip6.fr/ malouch/TR/sshcr.pdf.

[6] IEEE Std. 802.22 WRAN Part 22:Cognitive Wireless RAN Medium Access Control (MAC) and Physical Layer (PHY) Specifications: Policies and Procedures for Operation in the TV Bands, 2011. 